\title{
Physical Modeling Applies to Physiology, Too*
}

\author{
Vincent Hayward \\ Department of Electrical Engineering, \\ McGill University, Montréal, Québec H3A 2A7, Canada \\ hayward@mcrcim.mcgill. edu
}

\begin{abstract}
A physical model was utilized to show that the neural system can memorize a target position and is able to cause motor and sensory events that move the arm to a target with more accuracy. However, this cannot indicate in which coordinates the necessary computations are carried out. Turning off the lights causes the error to increase which is accomplished by cutting off one feedback path. The geometrical properties of arm kinematics and the properties of the kinesthetic and visual sensorial systems should be better known before inferences about higher levels of processing can be drawn.
\end{abstract}

An acceptable model for a physical system should be able to account for the observations in a wide range of situations and in a manner that does not depend on its own representation but only on that of the represented system. Coincidentally, this necessity is currently receiving a fair bit of attention in the robotics research, although, at least in the case of mechanical systems, the question was settled satisfactorily by the physicists and the mathematicians of the last century. Bruynincks (1991) has listed four types of invariance which are required to construct a theory with physical relevance. Although discussed in an engineering context, these invariances are also needed for physiological models. These are: invariance (1) to a change in reference frame, (2) to a change in physical units, (3) to a change in mathematical representation, and (4) to a change in arbitrary choices. It is unfortunate that the model proposed by Flanders et al. fails all four requirements. In other terms, one could construct uncountable variations of the proposed model that could equally explain the data.

The question of reference frame invariance is of course of direct relevance to the subject matter since the essence of the proposed model is to deal with coordinate changes. In the study and in this commentary, the human arm is

*in Behavioral Brain Sciences 1992 15(2):342-343, open peer commentary on Flanders, M., Tillery, S. I. H., Soechting, J. F. Early stages in a sensorimotor transformation. Behavioral Brain Sciences 1992 15(2):309-320. treated as three articulated rigid bodies: the torso used as the ground body, the arm and the forearm. Fig. 1 shows a cartoon version of the human arm kinematic arrangement in terms of two joints and three rigid links. For the sake of brevity, it will be discussed only intuitively. It can be readily observed that four coordinates are required to describe its position (and velocity). These coordinates can be chosen among many arbitrary possibilities. One fundamental reason from an engineering viewpoint, and most certainly, also from a physiological view point, for choosing one set rather than another is their respective computational advantages. From an engineering perspective, the reasons for making that choice are given by the computing hardware available to us. From a physiological perspective, the question cannot be so easily answered. The lack of detailed knowledge of how neural computations are carried out makes it difficult to speak of computational advantages other than in very general terms, if indeed it is a factor influencing Nature's choice. The discussion about coordinates is important because it directly affects that of reference frames. What has just been discussed may be illustrated by an example. In the case of computer control, the set

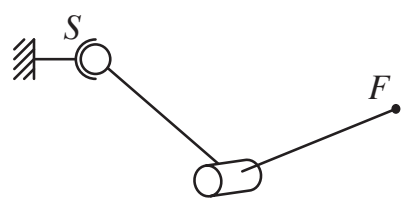

Figure 1: Grossly oversimplified kinematic geometry of upper limbs.

of coordinates for controlling mechanisms such as the one shown in Fig. 1, is rarely chosen to be minimal. In fact, one routinely selects the "redundant" rotation tensor to represent rotations on the grounds of convenience, numerical stability, and encoding simplicity. It would be surprising that Nature had not opted for a very high degree of redundancy in encoding coordinates. Generally speaking biological designs are characterized by stagger- 
ing levels of abundancy to implement devices, whether they are motor, sensor, or computational elements.

The choice of coordinates suggested in the paper in terms of arm and forearm elevation and yaw is less than minimal and presents many drawbacks. The representation to which they lead is arbitrary and no explanation is given for this choice. The model embodied by equations 2-5 depends on reference frames, physical units, and mathematical representations. Thus, all the conclusions that can be inferred from this model also depend on these factors. The spherical coordinates selected to encode the target positions suffer from the same problems, furthermore, they become undeterminate for locations which lie on the $z$ axis of the reference frame, thus causing subsequent transformations of these coordinates to break down. It can readily be observed that people can point their arms without coordinate transformation problems in a solid angle spanning more than half a sphere. So why should the brain be using these coordinates? The model also suffers from not being applicable to a wide range of situations. Another question is left in the dark by equations $2-5$. The model has three inputs and four outputs. On what grounds has a whole infinity of solutions been discarded? We shall return to this question later. Inspection of Fig. 8 is also puzzling. We could remove the label "Shoulder Centered" without changing the meaning of the diagram since all the other labels refer to angular quantities which do not depend on a particular origin.

The mechanism represented in Fig. 1 is meant to grossly approximate the arm kinematics. It has intrinsic properties which are completely independent from the mathematics that could be used to describe it. Without even invoking elementary notions of the theory of mechanisms, it can be appreciated upon inspection of the figure that when the elbow angle is fixed, the point F (symbolizing the finger) must move on the surface of sphere centered at $\mathrm{S}$ (the shoulder). Also, for a given position of point $\mathrm{F}$, there exists an infinity of possible arm configurations. As an aside, there exist rigorous techniques to carry out the analysis of mechanisms in general and of biomechanisms in particular, Screw Theory for example (Phillips 1986 and 1990). However, an intuitive account suffices for the purposes of this commentary.

Let us step back to section 2.2 of the paper. As a consequence of experimental evidence, it is stated that "the distance error did not depend on the values of elevation and azimuth". This can be explained by invoking independence of the kinesthetic perception of the elbow angle (which determines the distance) with respect to the shoulder posture. This does not tell us anything about coordinates, it is simply consistent with the sim- plified kinematic properties of the arm. The fact that the kinesthetic sense is more accurate for the shoulder joint than for the elbow joint and that the visual system can more uniformly appreciate angular position of targets than distances (Boff, Kauffman, and Thomas 1986) in conjunction with the arm geometrical properties furnish ample material to explain the data. Then, it is further stated that "the error is less consistent if evaluated from the head". Here, measurement results (the error) vary with the choice of reference frame. Surely, the model lacks an important property of invariance. What could that possibly tell us about which coordinates and reference frame(s) the CNS uses? Finally, how does the model explain that a unique finger tip position can be reached by an infinite number of distincts arm postures?

In summary, the fact that the neural system can memorize a target position and is capable to cause motor and sensory events that move the arm to a target with some accuracy cannot indicate in which coordinates the necessary computations are carried out. Cutting off one feedback path by turning off the lights causes the error to increase. This is to be expected given that the very purpose of feedback is to increase accuracy provided that sensors can make the relevant measurements. The geometrical properties of arm kinematics, the properties of the kinesthetic and visual sensorial systems should be better known before inferences about higher level of processing can be drawn, and finally physical modeling should be taken even more seriously in physiology than it is in engineering given the staggering complexity of the system under investigation.

\section{References}

Boff, K. R., Kaufman, L., Thomas, J. P. (1986) Handbook of Perception and Human Performance. John Wiley and Sons.

Bruynincks, H. (1991) Some invariance problems in robotics. Report 91R4, Dept. of Mechanical Eng., Katholieke Universiteit Leuven, Belgium.

Phillips J. 1986 and 1990. Freedom in Machinery. Vol. 1: Introducing screw theory, Vol. 2: Screw theory exemplified. Cambridge University Press. 